\title{
Theories of multiwavelength emission from Gamma-Ray Bursts: Prompt to afterglow
}

Soebur Razzaque ${ }^{* \dagger}$

Dept. of Physics, University of Johannesburg, PO Box 524, Auckland Park 2006, South Africa

E-mail: srazzaque@uj.ac.za

Gamma-Ray Bursts (GRBs) are powerful transient phenomena originating at cosmological distances. They outshine the entire gamma-ray sky for a brief period of time, less than 2 seconds for short-duration bursts and greater than 2 seconds for long- duration bursts. Non-thermal radiation from particles accelerated in shocks is widely thought to be the origin of GRBs. Initial prompt radiation, which is dominated by $\mathrm{keV}-\mathrm{MeV}$ gamma rays, is followed by fainter and long-lasting afterglow emission in gamma ray to radio wavelengths. In this article I will give an overview of observations and theoretical models for GRB emission from radio to very high-energy gamma rays, up to $100 \mathrm{GeV}$.

3rd Annual Conference on High Energy Astrophysics in Southern Africa-HEASA2015,

18-20 June 2015

University of Johannesburg, Auckland Park, South Africa

\footnotetext{
* Speaker.
}

†Invited talk. 


\section{Introduction}

A gamma-ray burst (GRB) is a bright flash of $\sim 10 \mathrm{keV}-1 \mathrm{MeV}$ gamma rays that lasts for $<2 \mathrm{~s}$ for a short GRB and $\geq 2 \mathrm{~s}$ for a long GRB. The typical gamma-ray flux for a long GRB is $F \approx(0.1-1) \times 10^{-5} \mathrm{erg} \mathrm{cm}^{-2} \mathrm{~s}^{-1}$, which implies an isotropic GRB luminosity $\gtrsim 10^{52} \mathrm{erg} \mathrm{s}^{-1}$ for a typical GRB redshift $z=1-3$. Thus GRBs are the most powerful transient phenomena in the universe. The observed rate of GRB is $\sim 1-2$ per day. The prompt gamma-ray emission from a GRB is typically followed by longer lasting, on the scales of hours-days-months, afterglow emission in gamma ray, X-ray, optical and radio. GRBs are therefore multiwavelength and multi-timescale astrophysical transients (see for reviews [1]).

It is widely believed from mounting evidence that long GRBs are endowed with deaths of massive stars [2]. Study of GRBs can therefore lead to understanding the explosion mechanism of massive stars, understanding link between spherical core-collapse supernova and jetted GRB explosions, star formation rate at high redshift, etc. GRBs, being the most powerful engines, have also been hypothesized as the sources of ultrahigh-energy cosmic rays (UHECRs) [3, 4] and have been predicted to be the sources of high-energy neutrinos [5]. Because of their cosmological distance and extreme luminosities in gamma rays with energy up to $\sim 100 \mathrm{GeV}$, GRBs have also been used as probes to constrain the intensity of infrared-ultraviolet background light in the universe which can produce electron-positron pairs by interacting with $\gtrsim 10 \mathrm{GeV}$ photons [6]. Various quantum gravity models, predicting energy-dependent light speed have been constrained as well from observations of GRBs.

In this brief review, we draw on historical to present-day GRB observations that have revealed various features of prompt and afterglow emission across the entire electromagnetic frequency range. We also discuss the fireball-shock model for the prompt and afterglow emission from GRBs and critically review the $\mathrm{GeV}$ emission mechanisms.

\section{Observations}

Historically GRBs were detected by the Vela 5-6 and A-B satellites in 1969-1979 as part of their monitoring of the Nuclear Test Ban treaty [7]. Although not designed for astronomy, these satellites detected 73 GRBs during this period. Scientific progress was slow, however, because of lack of pointing accuracy of the instruments and multiwavelength coverage. The breakthrough in GRB science came in the 1990's with the launch of the Compton Gamma-Ray Observatory (CGRO; 1991-2000). The Burst and Transient Source Experiment (BATSE) onboard the CGRO detected over 2500 GRBs [8]. Much of our knowledge on prompt emission, including short- and long-GRB classification, came from the BATSE GRBs [9]. The first afterglow was detected by BeppoSAX (1997-2002) in X-ray, which also enabled subsequent optical detections that require accurate GRB position [10, 11]. High Energy Transient Experiment (HETE) satellites (2000-2005) paved the path for current era of rapid localization of GRBs [12].

The NASA it Swift mission (2004- ) is the first modern autonomous repointing observatory dedicated to GRB detection [13]. it Swift has a wide-field imaging camera, Burst Alert Telescope (BAT), in the hard X-ray band that detects the bursts at a rate of $\sim 90$ GRBs per year. BAT provides arcminute position accuracy, which allows the spacecraft to autonomously and rapidly 
(100 s) reorient itself for sensitive X-ray and UV/optical observations of the afterglow using its X-Ray Telescope (XRT) and UV Optical Telescope (UVOT), respectively.

Launched in 2008, the NASA Fermi Gamma-ray Space Telescope has two wide-field instruments: the Large Area Telescope (LAT) is a pair conversion telescope covering the energy range from $20 \mathrm{MeV}$ to $>300 \mathrm{GeV}$ with a position accuracy of $\lesssim 1^{\circ}$ [14]. The Gamma-ray Burst Monitor (GBM) onboard the Fermi is composed of scintillation detectors covering $8 \mathrm{keV}$ to $40 \mathrm{MeV}$ [15]. GBM detects $\sim 300$ GRBs per year while LAT detects $\sim 10$ GRBs per year at relatively unexplored energy range of $\gtrsim 100 \mathrm{MeV}$ [16].

Satellite GRB triggers and positions are rapidly sent to the ground so that ground-based optical and radio telescopes can follow the afterglows. Many robotic optical telescopes such as MASTER (with one node in Sutherland, South Africa) and Watcher at the Boyden Observatory in Bloemfontein, South Africa, can autonomously point to the GRB positions acquired from satellites. The fraction of afterglows detected in X-ray, optical and radio is 30\% [17].

\subsection{Characteristics of prompt emission}

There are three characteristics of prompt emission, namely i) its duration $(<2 \mathrm{~s}$ for short GRBs and $\geq 2 \mathrm{~s}$ for long GRBs); ii) highly variable multi-peaked emission; and iii) non-thermal spectrum. The duration of a GRB is measured as the time scale during which $90 \%$ of the $\mathrm{keV}-\mathrm{MeV}$ gamma-ray fluence is acquired. The dichotomy of duration indicates that there are possibly two GRB progenitor classes. The flux variability of $\mathrm{keV}-\mathrm{MeV}$ emission has been measured often down to tens of milliseconds. Such short time scale variability indicates that the GRB central engine is very compact, of the order of the light-crossing length scale. The prompt gamma-ray spectra in the keV-MeV range are fitted with two power-laws smoothly connected with an exponential, the so-called Band function [18]. The non-thermal nature of the GRB spectra indicate particle acceleration at the emission sites.

The Fermi-LAT in recent years has discovered three new features of $\mathrm{GeV}$ emission from GRBs: i) a delayed onset compared to lower-energy emission detected by GBM; ii) a longer lasting emission compared to lower-energy bands; and iii) an emission component in addition to the Band spectra. These energy-dependent features have been observed in a number of GRBs, both long and short, for which adequate data are available [16]. Figure 1 shows light curves of the long GRB 080916C [19] in different energy bands as detected by the Fermi-GBM and Fermi-LAT with increasing energy from the top to the bottom panel. Note the delayed onset as well as longer lasting LAT, $>100 \mathrm{MeV}$, emission compared to the GBM emission. Figure 2 shows broad-band spectra of the long GRB 090926A which exhibits power-law (PL) and power-law with cutoff (CUTPL) in addition to the traditional Band spectrum [20].

\subsection{Characteristics of afterglow emission}

The prompt $\gamma$-ray emission of a GRB is followed by much longer-lasting afterglow emission, typically in X-ray, Ultraviolet, and optical wavelengths. Smooth, power-law decay of flux across the wavelengths is the main characteristic feature of the afterglow emission (see Fig. 3). Autonomous repointing capabilities of it Swift and Fermi has enabled continuous observation of GRBs in different wavelengths. it Swift-XRT in particular has followed evolutions from the prompt 


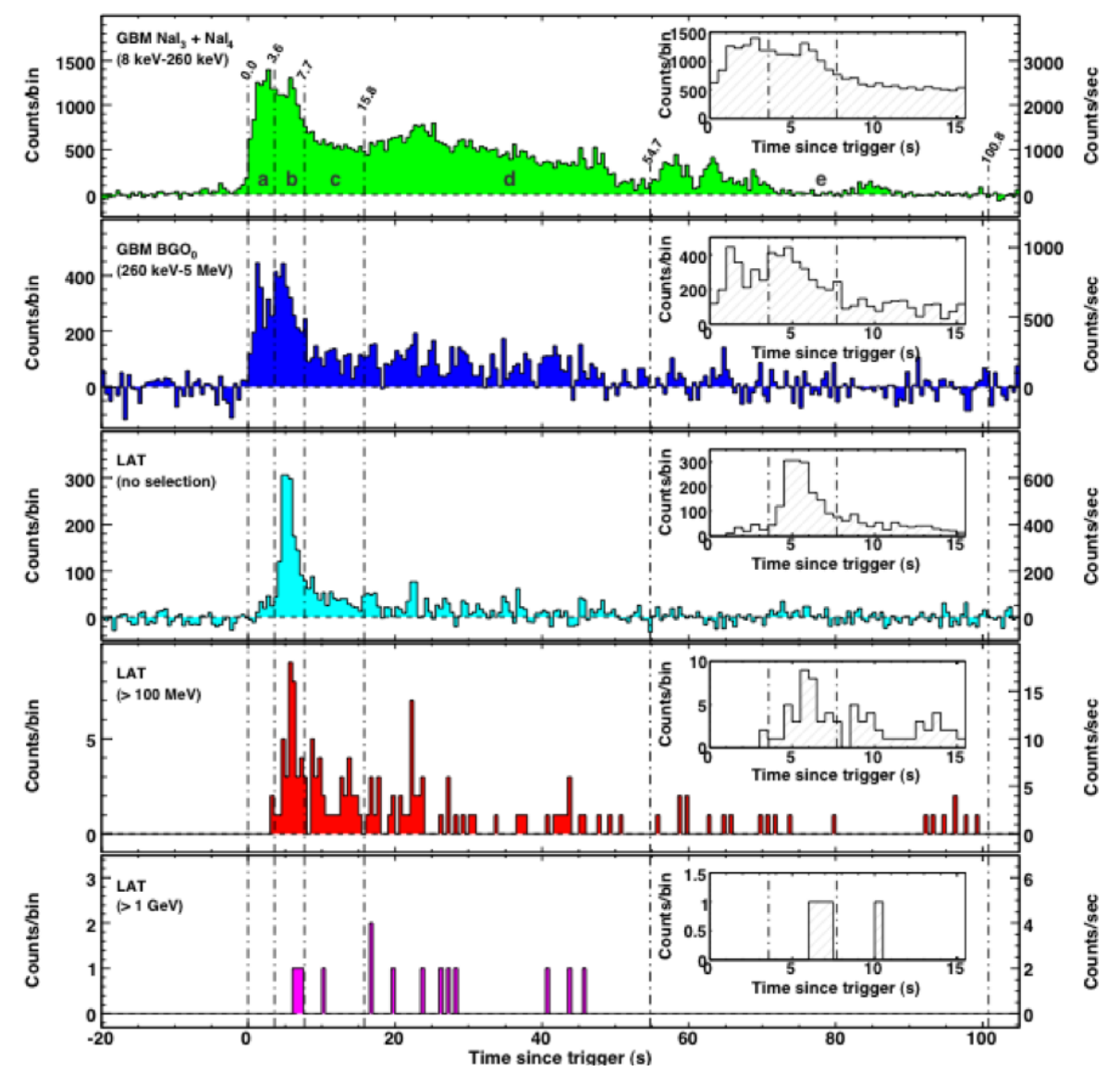

Figure 1: Photon count rates vs. time since trigger of GRB 080916c detected by Fermi-GBM (top three panels) and by Fermi-LAT (bottom two panels). Reproduced from Ref. [19].

to afterglow phases in X-ray, revealing complex nature of transition from the one phase to the other $[23,24]$. The highly-variable prompt emission is followed by a steep-decay phase $\left(F_{V} \propto t^{-3}\right)$ and often a shallow-decay phase $\left(F_{V} \propto t^{-1 / 2}\right)$ with occasional flares before the afterglow sets-in. Optical afterglow $\left(F_{V} \propto t^{-1}\right)$, which lasts for a few days, is crucial to obtain a GRB host-galaxy spectra to determine redshift as well as to probe the GRB jet-break time, when the edges of the highly collimated GRB jet become visible and the flux-decay becomes steeper $\left(F_{v} \propto t^{-2}\right)$ as a result. These in turn tell us about the total energetics of the burst.

Before the launch of Fermi, $\gamma$-ray emission was thought to be a part of the prompt emission only. Fermi-LAT detected $>100 \mathrm{MeV}$ emission from bright GRBs that extend far beyond the prompt emission detected by the Fermi-GBM. In case of the exceptionally bright burst GRB 130427A with GBM duration $\sim 300 \mathrm{~s}$, the $>100 \mathrm{MeV}$ emission lasted for about one day [22]. Figure 3 shows optical to $>100 \mathrm{MeV} \gamma$-ray lightcurves from GRB 110731A [21]. Simultaneous multiwavelength data from this burst allowed for the first time to conclude that temporally-extended $>100 \mathrm{MeV}$ Fermi-LAT emission is part of the GRB afterglow. 


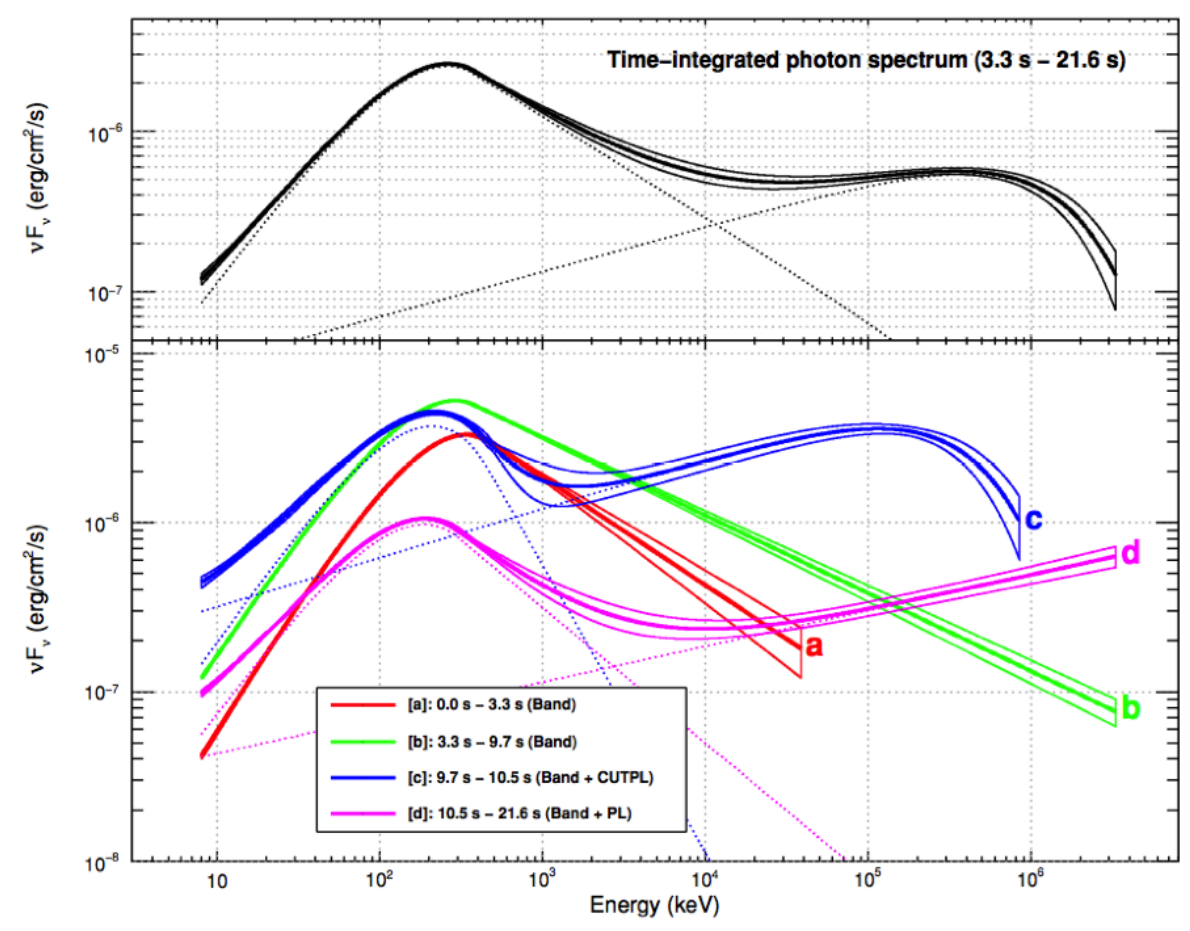

Figure 2: Time-integrated and time-resolved spectra of GRB 090926A. Reproduced from Ref. [20].

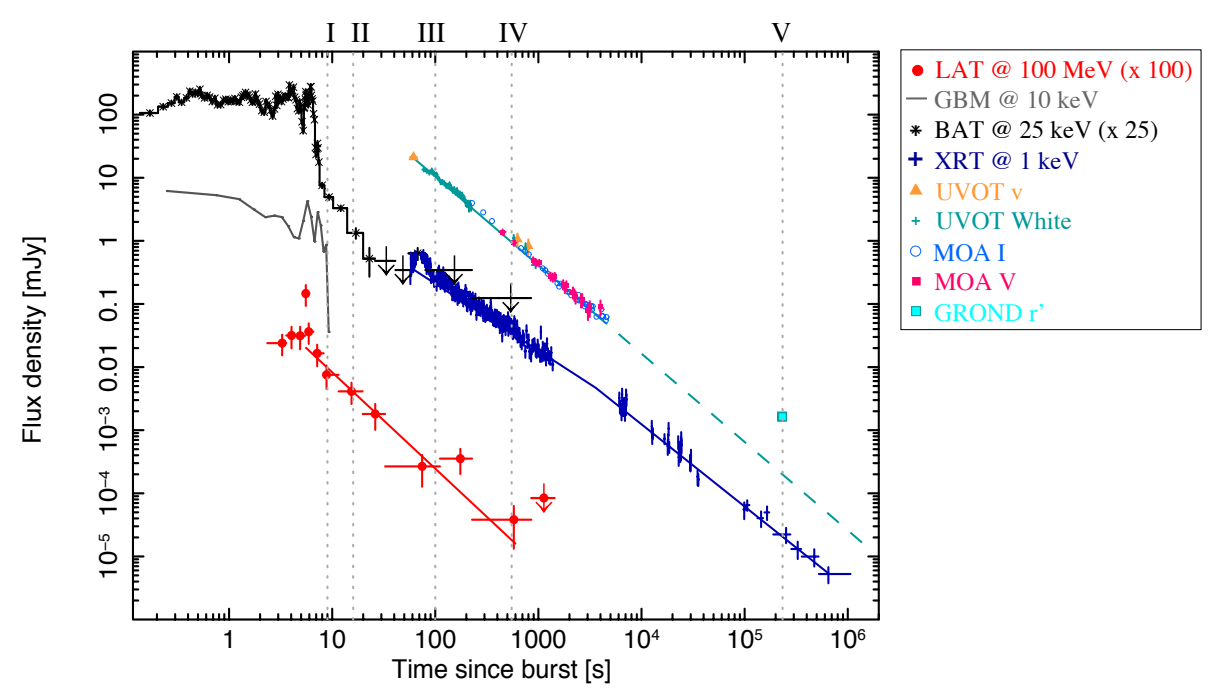

Figure 3: Optical, Ultraviolet, X-ray and $\gamma$-ray afterglow of GRB 110731A. Reproduced from Ref. [21]. 


\section{GRB fireball shock model}

Core-collapse of massive stars and binary mergers (neutron stars or neutron star-black hole) are thought to produce the long- and short-duration GRBs, respectively. A black hole or a magnetar and a short-lived accretion disc is formed in either case, with the launch of a relativistic jet, of which the exact mechanism is yet unknown. Isotropic-equivalent total energy outflow in the jet is $L_{0} \approx 10^{50}-10^{52} \mathrm{erg} / \mathrm{s}$ with an initial temperature $T_{0} \approx(1-10)\left(L_{0} / R_{0}^{2}\right)^{1 / 4} \mathrm{MeV}$ at the base of the jet with radius $R_{0} \approx 10^{6}-10^{7} \mathrm{~cm}$. Initially the jet contains gamma rays, electron-positron pairs and relatively fewer baryons (mostly protons). The jet expands due to the radiation pressure of an optically thick hot plasma [26]. The Lorentz factor of the bulk outflow increases with radius $R$ as $\Gamma \propto R / R_{0}$ until reaching a critical value $\eta=L_{0} / \dot{M} c^{2} \sim 100-1000$, called the baryon-loading parameter, where $\dot{M}$ is the mass outflow rate in the jet. Kinetic energy in the jet, mostly in baryons, dominates over the thermal energy at $\Gamma \approx \eta$ and the jet coasts with constant $\Gamma$ afterwards [27].

\subsection{Prompt emission from internal shocks}

The observed prompt keV-MeV $\gamma$-ray emission from GRBs is widely believed to be due to collisions between shells of plasma, producing shocks (internal shocks) that convert a significant fraction of the fireball kintetic energy into radiation energy [32]. Internal shocks take place over a wide range of distances from the GRB central engine, $r_{i} \sim \Gamma^{2} c t_{v} \sim 3 \times 10^{13}(\Gamma / 316)^{2}\left(t_{v} / 0.01 \mathrm{~s}\right) \mathrm{cm}$, where $t_{v} \sim 0.001-1 \mathrm{~s}$ is the flux variation time and $\Gamma \sim 10^{2}-10^{3}$ is the bulk Lorentz factor of the shocked shells. Although the exact radiation mechanism is unknown, synchrotron radiation from relativistic electrons accelerated in the internal shocks is currently the most favorite model.

An estimate of the magnetic field in the jet frame is typically inferred from the prompt $\gamma$-ray observations as $B^{\prime}=\left(2 \varepsilon_{B} L_{\gamma, i s o} / \varepsilon_{e} r_{i}^{2} \Gamma^{2} c\right)^{1 / 2}$, where $\varepsilon_{e}$ and $\varepsilon_{B}$ are equipartition parameters denoting the fraction of the shock energy in the electrons and magnetic fields, respectively. For typical parameter values $B^{\prime} \sim 2 \times 10^{4}\left(\varepsilon_{B} / \varepsilon_{e}\right)^{1 / 2}\left(L_{\gamma, \text { iso }} / 10^{51} \mathrm{erg} \mathrm{s}^{-1}\right)^{1 / 2}(\Gamma / 316)^{-3}\left(t_{v} / 0.01 \mathrm{~s}\right)^{-1} \mathrm{G}$ [28]. Electrons with a minimum Lorentz factor, $\gamma_{e}^{\prime} \sim m_{p} / m_{e}$, can explain the observed $\varepsilon_{p k} \sim 100 \mathrm{keV}-1 \mathrm{MeV}$, the typical $v F_{v}$ peak of the $\gamma$-ray spectrum in coincidence with the characteristic synchrotron radiation peak. At energies above $\varepsilon_{p k}$, the spectrum can be explained as due to synchrotron radiation from the power-law distributed electron Lorentz factor, $n\left(\gamma_{e}^{\prime}\right) \sim \gamma_{e}^{\prime-p}$, as $n(\varepsilon) \sim \varepsilon^{-p / 2-1}$.

Observation of an additional spectral component (see Fig. 2), in the form of a power-law that domintes $\gtrsim 100 \mathrm{MeV}$ emission, from a number of Fermi-LAT GRBs has led to modelling radiation processes other than synchrotron radiation by relativistic electrons. Synchrotron-SelfCompton (SSC) scattering of soft target photons by relativistic electrons in the internal shocks is an energetically frugal model $[33,34,35,30]$, however, it is difficult to explain a delayed onset of the $\gtrsim 100 \mathrm{MeV}$ emission compared to the $\mathrm{keV}-\mathrm{MeV}$ emission (see Fig. 1). Radiation originating from protons and nuclei has been considered as another mechanism to produce the additional spectral component in the prompt phase. These models are proton-synchrotron radiation and synchrotron radiation from $\gamma \gamma \rightarrow e^{+} e^{-}$pairs [36] (see Fig. 4); photohadronic ( $p \gamma$ ) interactions and associated cascade radiation [37, 38]; and photodisintegration of heavy nuclei in the GRB jet [39] (see Fig. 5). The total energy of the GRB jet needs to be $\sim 10-1000$ times the observed $\gamma$-ray energy in these hadronic models. 


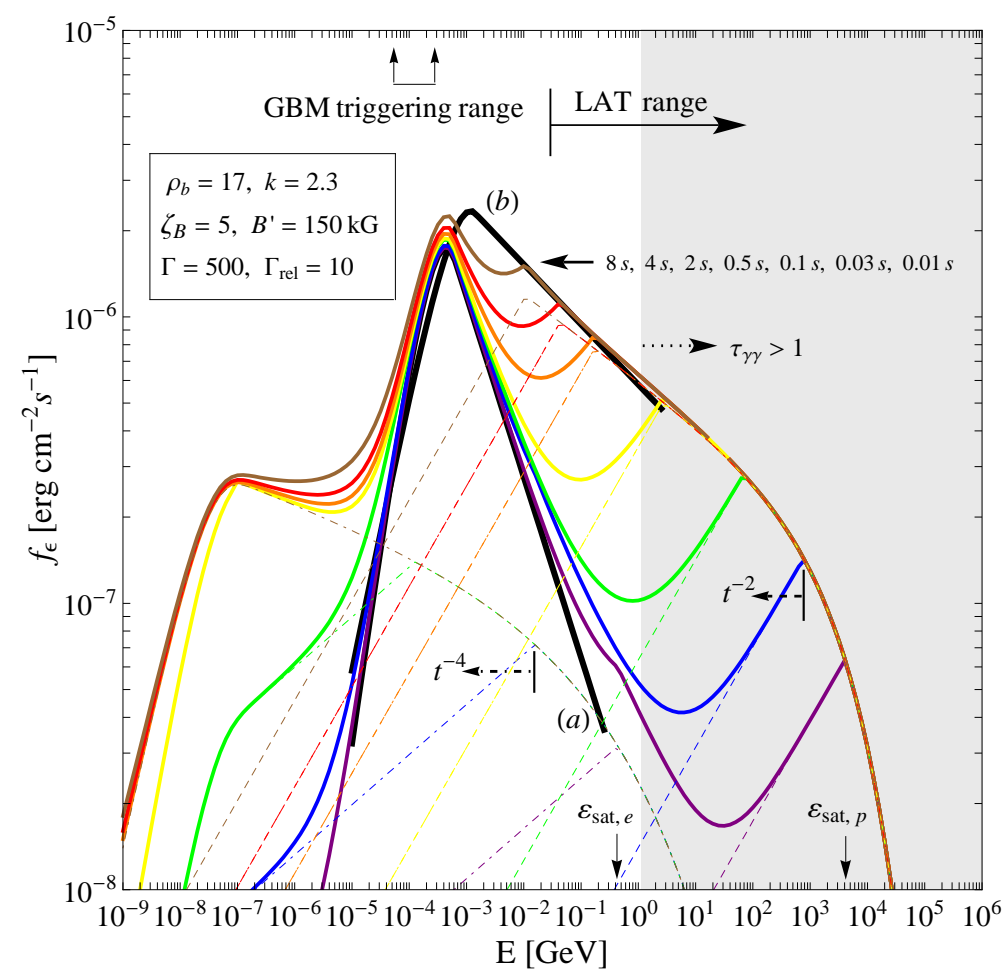

Figure 4: Synchrotron radiation from protons for the delayed onset of LAT emission in GRB 080916C. The delay is due to build-up of proton-synchrotron flux and a shift of cooling frequency from very high energies to the Fermi-LAT energy range, with time. Reproduced from [36].

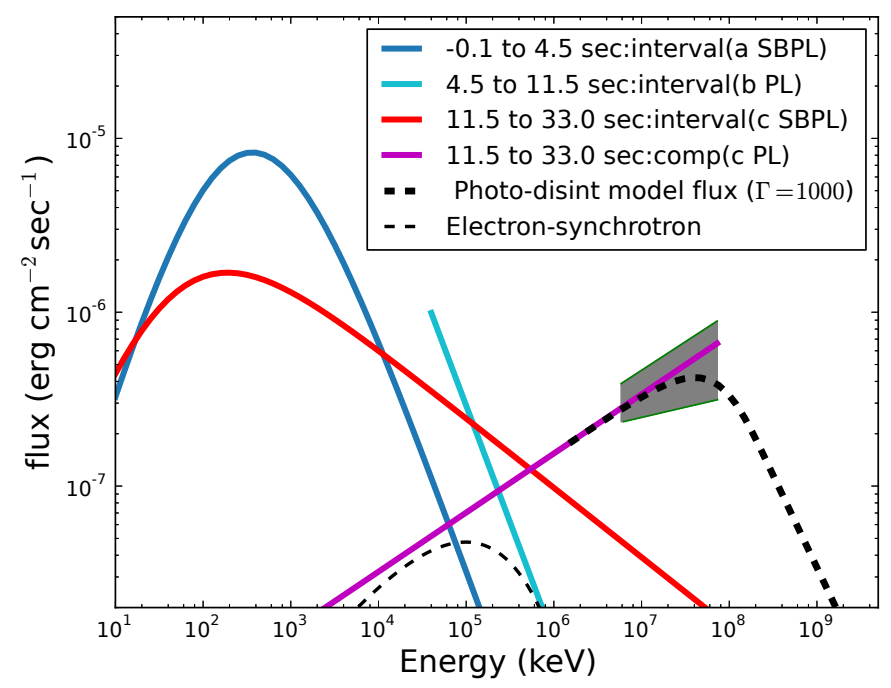

Figure 5: Photodisintegration of Iron nuclei in the jet of GRB 130427A to produce the power-law radiation component (thick, black dashed curve) in the prompt phase. Reproduced from Ref. [39]. 


\subsection{Afterglow emission from external shocks}

The long-lasting afterglow, characterized by smoothly decaying flux, is due to emission from a GRB blast-wave decelerating in a self-similar fashion [40]. A forward shock and a reverse shock (external shocks) are formed when the GRB jet slows down by accumulating circumburst material. This slow down happens when the jet kinetic energy is comparable to the kinetic energy of the swept-up material in the blast wave, $E_{i s o}=(4 / 3) \pi r_{d e c}^{3} n_{e x t} m_{p} c^{2} \Gamma^{2}$. The characteristic slow down radius is $r_{\text {dec }} \sim 2 \times 10^{17}\left(E_{\text {iso }} / 10^{53} \mathrm{erg}\right)^{1 / 2}\left(n_{\text {ext }} / 1 \mathrm{~cm}^{3}\right)^{-1 / 2}(\Gamma / 100)^{2 / 3} \mathrm{~cm}$, for a constant density $\left(n_{\text {ext }}\right)$ interstellar medium. In the self-similar phase, the bulk Lorentz factor of the blast-wave evolves as $\Gamma \sim t^{-3 / 8}$ for an adiabatic fieball and $\Gamma \sim t^{-3 / 7}$ for a radiative fireball, both in a constant density medium $[40,41]$.

Synchrotron radiation from relativistic electrons in the external forward shock is used to model radio-to-optical and X-ray afterglow $[42,41]$. As the blast-wave continues to decelerate by sweeping up more and more external matterial, the peak of the synchrotron flux shifts from the highfrequency to low-frequency bands. Smooth decay of the flux across different energy bands is a characteristic feature of the afterglow synchrotron model [43], whose predictions allowed first measurements of the GRB host galaxies and redshifts [10,11]. The external shocks electronsynchrotron modelling of the broad-band afterglow emission has proven to work in a good sample of GRBs.

Gamma-ray emission from external shocks was not widely discussed before Fermi-LAT convincingly detected $\gtrsim 100 \mathrm{MeV}$ emission that lasted much longer than the prompt keV-MeV emission. In case of the record-breaking GRB 130427A, the $\gtrsim 100 \mathrm{MeV}$ emission lasted for 24 hours [22]. Long duration with smooth flux decay (see Fig. 3) and delayed onset observed in a number of bright Fermi-LAT GRBs led interpretation of high-energy radiation from the external forward shock [44, 45, 46, 47, 48]. The delayed onset of the LAT emission is explained as the time required for deceleration of the GRB ejecta with high bulk Lorentz factor.

Simultaneous observations of GRB 110731A from optical to $\gamma$-rays allowed the first opportunity to test the afterglow model for the temporally extended $\gtrsim 100 \mathrm{MeV}$ emission. Afterglow model of electron-synchrotron radiation from the forward shocks of decelerating blast wave in an wind environment fits the multwavelength light curves (see Fig. 3) and broadband SEDs at different epochs (see Fig. 7) rather well. The onset of the afterglow at $\sim 9 \mathrm{~s}$ after the trigger is also consistent with a high bulk Lorentz factor of $\sim 500$.

Detection of $\mathrm{GeV}$ photons long after the triggers of a number of GRBs, however, poses a challenge to the simple electron-synchrotron radiation for these photons. GRB 130427A imposes the most stringent constraint [22]. Proton-synchrotron radiation [47] and or inverse-Compton scattering [49] could potentially alleviate this issue with an additional radiation component.

\section{Outlook}

Multi-wavelength observations of GRBs are crucial to understand explosive death of massive stars and emission mechanisms. Detection of long-lived high-energy emission up to $100 \mathrm{GeV}$ is very promising for ground-based Cherenkov telescopes. Prompt follow-up strategy is needed to be in place for GRB study. A South African GRB community can greatly enhance use of the radio to gamma-ray observatories in the Southern Hemisphere. 


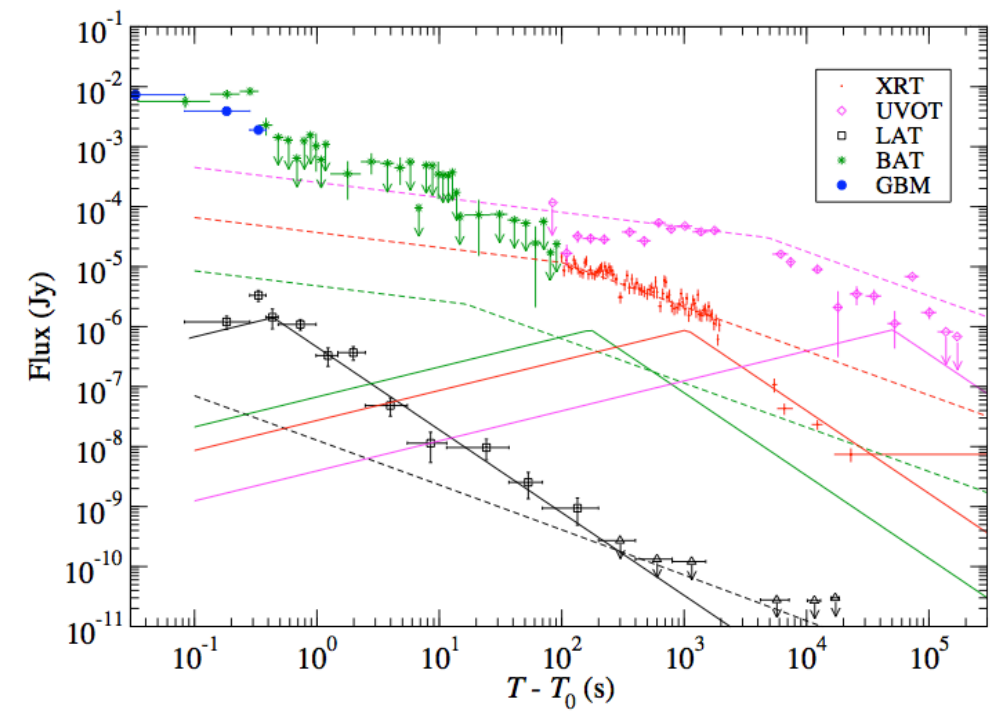

Figure 6: Modelling of multiwavelength light curves of GRB 090510 using synchrotron radiation from electrons and protons in a decelrating blast wave in a constant density medium. The LAT data is fitted by proton-synchrotron radiation. Reproduced from Ref. [47].

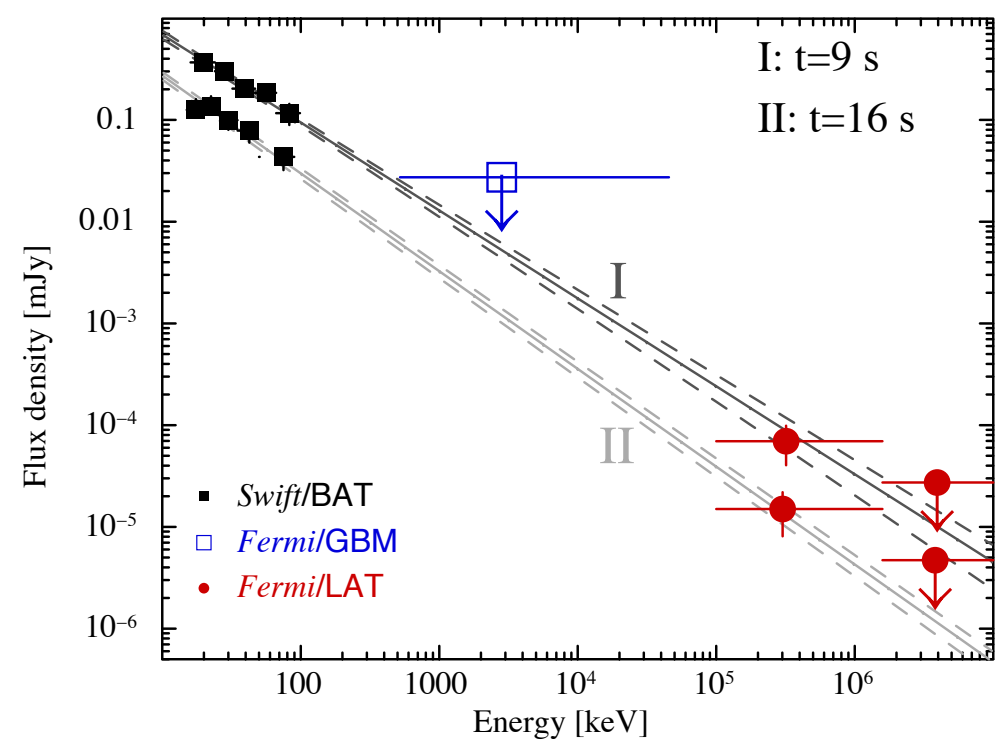

Figure 7: Broadband SED of GRB 110731A in two very early time bins and afterglow model fits. Reproduced from Ref. [21]. 


\section{Acknowledgments}

Supports from the South African Gamma-ray Astronomy Programme (SA-GAMMA) and the National Research Foundation (NRF) of South Africa are gratefully acknowledged.

\section{References}

[1] N. Gehrels and S. Razzaque, Front. Phys. China 8, 661 (2013) doi:10.1007/s11467-013-0282-3 [arXiv:1301.0840 [astro-ph.HE]].

[2] K. Z. Stanek et al., Astrophys. J. 591, L17 (2003) doi:10.1086/376976 [astro-ph/0304173].

[3] E. Waxman, Phys. Rev. Lett. 75, 386 (1995) doi:10.1103/PhysRevLett.75.386 [astro-ph/9505082].

[4] M. Vietri, Astrophys. J. 453 (1995) 883 doi:10.1086/176448 [astro-ph/9506081].

[5] E. Waxman and J. N. Bahcall, Phys. Rev. Lett. 78, 2292 (1997) doi:10.1103/PhysRevLett.78.2292 [astro-ph/9701231].

[6] A. A. Abdo et al. [GBM Collaboration], Astrophys. J. 723, 1082 (2010) doi:10.1088/0004-637X/723/2/1082 [arXiv:1005.0996 [astro-ph.HE]].

[7] R. W. Klebesadel, et al., Astrophys. J. 182, L85 (1973)

[8] C. A. Meegan, et al., Nature 355, 143 (1992)

[9] C. Kouveliotou, et al., Astrophs. J. 413, 101 (1993)

[10] E. Costa, et al., Nature 387, 783 (1997)

[11] J. van Paradijs, et al., Nature 386, 686 (1997)

[12] D. Q. Lamb et al., New Astron. Rev. 48, 423 (2004) [Nucl. Phys. Proc. Suppl. 132, 279 (2004)] [astro-ph/0309462].

[13] N. Gehrels, et al., Astrophys. J. 611, 1005 (2004)

[14] W. B. Atwood, et al., Astrophys. J. 697, 1071 (2009)

[15] C. Meegan, et al., Astrophys. J. 702, 791 (2009)

[16] M. Ackermann, M. Ajello et al. [Fermi-LAT Collaboration], Astrophys. J. Suppl. 209, 11 (2013) [arXiv:1303.2908 [astro-ph.HE]].

[17] P. Chandra and D. A. Frail, Astrophys. J. 746, 156 (2012) doi:10.1088/0004-637X/746/2/156 [arXiv:1110.4124 [astro-ph.CO]].

[18] D. Band et al., Astrophys. J. 413, 281 (1993). doi:10.1086/172995

[19] A. A. Abdo et al. [Fermi-LAT Collaboration], Science, 323, 1688 (2009)

[20] M. Ackermann et al. [Fermi-LAT Collaboration], Astrophys. J. 729, 114 (2011)

[21] M. Ackermann et al. [Fermi-LAT Collaboration], Astrophys. J. 763, 71 (2013)

[22] M. Ackermann et al. [Fermi-LAT Collaboration], Science, 343, 42 (2014)

[23] J. A. Nousek, et al., Astrophys. J. 642, 389 (2006)

[24] B. Zhang, et al., Astrophys. J. 642, 354 (2006)

[25] . Ackermann et al. [Fermi-LAT Collaboration], Science 343, 42 (2014) 
[26] P. Mészáros, P. Laguna and M. J. Rees, Astrophys. J. 415, 181 (1993)

[27] P. Mészáros and M. J. Rees, Astrophys. J. 530, 292 (2000)

[28] S. Razzaque, P. Mészáros and B. Zhang, Astrophys. J. 613, 1072 (2004) [astro-ph/0404076].

[29] S. Razzaque and P. Mészáros, Astrophys. J. 650, 998 (2006) [astro-ph/0601652].

[30] M. Ackermann, et al., Astrophys. J. 716, 1178 (2010)

[31] A. A. Abdo, et al., Astrophys. J. Lett. 706, L138 (2009)

[32] M. J. Rees and P. Mészáros, Astrophys. J. 430, L93 (1994) [astro-ph/9404038].

[33] X. -Y. Wang, Z. Li, Z. -G. Dai and P. Mészáros, Astrophys. J. 698, L98 (2009) [arXiv:0903.2086 [astro-ph.HE]].

[34] Z. Bosnjak, F. Daigne and G. Dubus, Astron Astrophys. 498, 677 (2009) [arXiv:0811.2956 [astro-ph]].

[35] K. Toma, X. -F. Wu and P. Mészáros, Astrophys. J. 707, 1404 (2009) [arXiv:0905.1697 [astro-ph.HE]].

[36] S. Razzaque, C. D. Dermer and J. D. Finke, Open Astron. J. 3, 150 (2010) [arXiv:0908.0513 [astro-ph.HE]].

[37] K. Asano, S. Guiriec and P. Mészáros, Astrophys. J. 705, L191 (2009) [arXiv:0909.0306 [astro-ph.HE]].

[38] K. Murase, K. Asano, T. Terasawa and P. Mészáros, Astrophys. J. 746, 164 (2012) [arXiv:1107.5575 [astro-ph.HE]].

[39] J. C. Joshi, S. Razzaque and R. Moharana, MNRAS Lett. (in press) [arXiv:1512.02434 [astro-ph.HE]].

[40] R. D. Blandford \& C. F. McKee, Physics of Fluids, 19, 1130 (1976)

[41] R. Sari, T. Piran \& R. Narayan, Astrophys. J. Lett. 497, L17 (1998)

[42] P. Mészáros and M. J. Rees, Astrophys. J. 476, 232 (1997) [astro-ph/9606043].

[43] R. A. M. J. Wijers, M. J. Rees and P. Mészáros, Mon. Not. Roy. Astron. Soc. 288, L51 (1997) [astro-ph/9704153].

[44] P. Kumar and R. B. Duran, Mon. Not. Roy. Astron. Soc. 409, 226 (2010) [arXiv:0910.5726 [astro-ph.HE]].

[45] G. Ghisellini, G. Ghirlanda, L. Nava \& Celotti, A. MNRAS 403, 926 (2010)

[46] M. De Pasquale, et al., Astrophys. J. Lett. 709, L146 (2010)

[47] S. Razzaque, Astrophys. J. Lett. 724, L109 (2010) [arXiv:1004.3330 [astro-ph.HE]]

[48] A. Corsi, D. Guetta and L. Piro, Astrophys. J. 720, 1008 (2010) [arXiv:0911.4453 [astro-ph.CO]].

[49] B. Zhang and P. Meszaros, Astrophys. J. 559, 110 (2001) [astro-ph/0103229]. 